Randomized Control Trial

\title{
Transforaminal Versus Lateral Parasagittal Versus Midline Interlaminar Lumbar Epidural Steroid Injection for Management of Unilateral Radicular Lumbar Pain: A Randomized Double- Blind Trial
}

Jeetinder Kaur Makkar, MD1, Kalla Krishna Prasad Gourav, MD', Kajal Jain, MD1, Preet Mohinder Singh, MD², Sarvdeep S. Dhatt, $\mathrm{MS}^{3}$, Naresh Sachdeva, MD ${ }^{4}$, and Sanjay Bhadada, $\mathrm{MD}^{4}$

\footnotetext{
From: ${ }^{1}$ Department of Anesthesia and Intensive Care, Post Graduate Institute of Medical Education and Research, Chandigarh, India; ${ }^{2}$ Department of Anesthesia, All India Institute of Medical

Sciences, New Delhi, India; 3Department of Orthopedics,

Post Graduate Institute

of Medical Education and Research, Chandigarh, India; 4Department of Endocrinology, Post Graduate Institute of Medical Education and Research, Chandigarh, India

Address Correspondence: Jeetinder Kaur Makkar, MD Department of Anesthesia and Intensive Care, Post Graduate Institute of Medical Education and Research $447 / 2$, Sector $45 \mathrm{~A}$ Chandigarh, 160 147, India E-mail: jeet1516@gmail.com

Disclaimer: There was no external funding in the preparation of this manuscript. Conflict of interest: Each author

certifies that he or she, or a member of his or her immediate

family, has no commercial association (i.e., consultancies, stock ownership, equity interest, patent/licensing arrangements, etc.) that might pose a conflict of interest in connection with the submitted manuscript.

Manuscript received: 02-05-2018 Revised manuscript received: 05-08-2019

Accepted for publication: 05-16-2019

Free full manuscript: www.painphysicianjournal.com
}

Background: Epidural steroid injections (ESIs) are commonly used for management of lumbosacral radicular pain. Midline interlaminar (MIL) or transforaminal (TF) routes are commonly used. The TF route, although associated with higher delivery of drug to the ventral epidural space, has serious complications including spinal cord injury and permanent paralysis reported in literature. Therefore, there is a search for a technically better route with fewer complications and greater drug delivery into the ventral epidural space. Recently, a parasagittal interlaminar (PIL) approach has been defined.

Objectives: We conducted this study to compare therapeutic effectiveness of 3 techniques of ESIs in patients having unilateral lumbar radiculopathy. Further, effect of ESI on bone mineral density (BMD) and serum osteocalcin levels were studied.

Study Design: Randomized double-blind trial.

Setting: Pain clinic of a tertiary care hospital.

Methods: Sixty-five patients were randomly allocated into group MIL, group PIL, and group TF to receive epidural injection with $80 \mathrm{mg}$ of methylprednisolone and $2 \mathrm{~mL}$ of $2 \%$ lidocaine. Effective pain relief and improvement in disability were assessed using Visual Analog Scale (VAS) and Modified Oswestry Disability Questionnaire (MODQ) scores at 2 weeks, 4 weeks, 3 months, and 6 months, respectively. Patients with $<50 \%$ relief received additional injection. Primary outcome of study was effective pain relief at 6 months. Mean change in VAS and MODQ scores, BMD, and serum osteocalcin levels were secondary outcome assessed.

Results: Patients having effective pain relief were significantly higher in group PIL (16 of 20 [80\%]) and group TF (15 of 20 [75\%]) compared with group MIL. Patients receiving ESI in group $\mathrm{PIL}$ and group TF showed significantly lower VAS scores than group MIL $(P=0.02, P=0.50$ at 3 months and $P=0.00, P=0.02$ at 6 months, respectively). Mean MODQ scores in group PIL and group TF were significantly lower than group MIL. However, group PIL and group TF did not significantly differ in MODQ scores. There was no significant change in serum osteocalcin and BMD, as assessed by dual energy $x$-ray absorptiometry scan at 3 months.

Limitations: The absence of a placebo control group, small sample size, and relatively short follow-up of 6 months were limitations.

Conclusions: PIL approach is equivalent to TF and superior to MIL approach in terms of effective pain relief and decrease in disability in patients with unilateral lumbar radiculopathy. This study showed no deleterious effect on BMD.

Key words: Epidural steroid, technique, efficacy, bone marrow density, serum osteocalcin

Pain Physician 2019: 22:561-573 
umbar disc herniation is a common cause of low back pain (LBP) with radicular leg pain (1-3). LBP can be treated using various modalities such as conservative management, epidural steroid injections (ESIs), and surgical interventions. Conservative options begin with neuropathic medications, topical modalities, or physical therapies. ESIs are offered when there is no improvement in pain relief after conservative treatments $(4,5)$. Many studies have shown that large amounts of phospholipase A2 produced due to disc herniation enhances production of prostaglandins leading to inflammation and pain $(6,7)$. This inflammatory response is reduced by ESIs, either by inhibiting the synthesis or release of proinflammatory substances (8).

Epidural space is usually approached using the interlaminar (IL), caudal, or transforaminal (TF) routes (9). Effectiveness of ESIs for pain relief depends on delivery of drug close to the site of pathology (9-13). It is probably because of this reason that the TF technique is associated with greater effectiveness. However, major complications such as spinal cord injury and paraplegia owing to embolization of the artery of Adamkiewicz has been reported with the technique (14-15). Infarction of the spinal cord with TF injection continues to be reported despite negative aspiration of blood and the use of continuous digital subtraction fluoroscopy (16).

Using the IL route, posterior epidural space can be approached between the adjacent spinous process, that is midline interlaminar (MIL), or the lateral most part of the lamina, that is parasagittal interlaminar (PIL) $(11,17)$. Limited effectiveness of MIL ESI has been reported in various studies $(9,11)$. This may be owing to a limited spread of drug to the ventral epidural space $(9,11)$. Current data suggests that the PIL route has a greater effectiveness than the MIL route in terms of ventral spread of the drug, and thereby better pain relief (11).

To date, no randomized clinical trials (RCTs) have compared TF, PIL, and MIL techniques for assessing the effectiveness of ESI in controlling pain in patients with chronic unilateral radicular pain. Therefore, the present study was undertaken to compare the therapeutic effectiveness of these 3 routes for administering ESI in patients with unilateral radicular LBP that has not responded to conservative treatment. Because use of a neuraxial steroid may induce osteoporosis secondary to increased bone resorption and decreased bone formation, effect of steroid given epidurally on bone mineral density (BMD) was also evaluated using dual energy x-ray absorptiometry (DEXA) scan $(18,19)$. We hypothesized that lateral PIL approach would be associated with better clinical outcome as compared with the other 2 approaches. The primary aim of the study was to define the incidence of effective pain relief at 6 months following ESI. Other secondary aims measured were mean change in Visual Analog Scale (VAS) and Modified Oswestry Disability Questionnaire (MODQ) scores over a period of 6 months, contrast medium spread pattern on fluoroscopy, number of ESIs required to achieve VAS score $<50 \%$ of baseline, and BMD using DEXA scan at baseline and at 3 months after ESI. Other markers of BMD such as serum osteocalcin levels, serum calcium levels, and vitamin D3 levels at baseline and at 3 months after first ESI were also measured.

\section{Methods}

\section{Study Design}

The study was a prospective, single-center, randomized, double-blind, active-controlled clinical trial. It was conducted in accordance with the Consolidated Standards of Reporting Trials (CONSORT) guidelines (20) and followed the principles of the Declaration of Helsinki. The institutional review board of the Post Graduate Institute of Medical Education and Research, Chandigarh, India approved study protocol and all patients provided written informed consent. Registration with the Clinical trial registry-India occurred on January 12, 2016 (registration number 2016/01/006514).

\section{Patients}

Adult patients of either gender between the age group of 20 and 50 years, with a diagnosis of chronic lumbar back pain and a unilateral radicular component for at least a 3-month duration not responding to conservative therapies, and having a pain score of at least 50 as assessed on 0 to 100 VAS at baseline were eligible for study recruitment. All patients underwent BMD evaluation, and only those with a $\mathrm{T}$ score +1.0 or more of total hip and spine was enrolled. Magnetic resonance imaging was performed to correlate level of herniation with symptoms of patient.

Patients who had surgery on the lumbar spine in the past, lumbar canal stenosis, spondylolisthesis, facet joint arthropathy, allergy to contrast medium or steroid, and bleeding diathesis were excluded. Patients with a history of systemic steroid use, use of 
lumbar ESI in the past 6 months, or a history of any disorder known to affect the bone turnover were also excluded.

\section{Randomization and Blinding}

Randomization was performed by an independent pharmacist using a computer generated randomization schedule (Software Research Randomizer (Urbaniak, GC \& Plous S 2013, version 4.0), blocks of 6). Random numbers were kept in opaque, sealed envelopes and opened by an independent anesthesiologist at the time of injection. None of the study investigators, including the outcome assessor, had access to the randomization sequence. ESIs were performed using one of the 3 approaches: group MIL ESI using midline IL approach, group PIL ESI using PIL approach, group TF ESI using TF approach.

All procedures were performed by a single investigator (J.K.M.) and followed by other investigators (B.G. and K.P.G.). Study cases were kept in between clinical nonstudy cases during the procedure and for follow-up. This was done to enhance the blinding and allocation concealment. Both patients and the investigator assessing the patient were unaware of the group allocation.

\section{Study Intervention and Procedure}

After application of standard monitoring, patients were placed in the prone position. Level of intervention was determined by clinical presentation and confirmed using magnetic resonance imaging findings. An initial anteroposterior fluoroscopic image was obtained, and level of intervention identified.

In the MIL group, skin at target site was infiltrated with $2 \%$ lidocaine. An 18-gauge, 3.5-inch Tuohy needle was introduced at the midpoint between 2 spinous processes and epidural space identified using the lossof-resistance to saline solution technique. Final position of needle in-between the 2 spinous processes was confirmed with fluoroscopy before giving contrast medium.

In the PIL group, the needle was introduced into the most lateral part of the epidural space at target level, and advanced from posterior to anterior direction. Parasagittal orientation of the needle was maintained throughout the procedure.

In the TF group, fluoroscope was rotated through 15 to 20 degrees to get an ipsilateral oblique view until the superior articular process of the infrasegmental level was seen at the 6 o'clock position of the target pedicle. The overlying soft tissue was anesthetized with $1 \%$ lidocaine. A 22-gauge spinal needle was then advanced into the "safe triangle," inferior to the pedicle and superolateral to the exiting spinal nerve. Final needle tip was verified by fluoroscopy as caudad to the pedicle shadow in anteroposterior and mid or ventral aspect of foramen in lateral views.

After negative aspiration, $0.5 \mathrm{~mL}$ lohexol $(300 \mathrm{mg} /$ $\mathrm{mL}$ ) (Omnipaque, GE Healthcare, London, United Kingdom) was injected to confirm placement of the needle in the epidural space. Contrast medium $(3.5 \mathrm{~mL})$ was then further injected to record the pattern of spread of the contrast medium, and to exclude any intravascular, subarachnoid, subdural, or intradiscal spread. Contrast medium was injected under continuous fluoroscopy in the TF group. Nerve root delineation was confirmed in the TF group.

Lateral images were used to evaluate ventral epidural spread. Ventral spread was present if contrast medium was seen hugging the posterior aspect of the contiguous vertebral body at or above the level of needle insertion in the lateral image. The perineural spread was defined as nerve root infiltration of contrast medium. Extent of spread of contrast medium was defined in terms of caudal and cephalic segments travelled by the contrast medium.

A solution of $80 \mathrm{mg}$ of methylprednisolone acetate (Depo-Medrol injection, Pfizer Products India Pvt Ltd, Mumbai, India) with $2 \mathrm{~mL}$ of $1 \%$ lidocaine (total volume $4 \mathrm{~mL}$ ) was then injected.

\section{Postintervention}

Patients were assessed for neurologic complication including postural headache, motor weakness, newly developed pain, paraplegia, and paresthesia.

\section{Follow-Up}

All patients were followed for a period of 6 months in the pain clinic by a blinded investigator (B.G. and K.P.G.) who was not aware of the technique used for performing ESI. Pain relief was recorded using VAS ranging from 0 to 10 and disability and impairment using MODQ at an interval of 2 weeks, 4 weeks, 3 months, and 6 months. Patients who reported $50 \%$ or less pain relief from baseline after ESI received additional injection with the same approach at least 15 days apart with a maximum of 3 injections. Those who reported $>50 \%$ pain relief received further ESIs only if pain increased to $50 \%$ of baseline again. In patients who developed $>50 \%$ pain relief compared with the baseline, the intervention was defined to be successful.

All patients underwent DEXA scan for BMD at time of enrollment and at 3 months. 
Serum calcium, vitamin D3, and serum osteocalcin were evaluated at baseline and at 3 months.

\section{Cointerventions}

All patients continued to receive conservative management (pregabalin/gabapentin, nonsteroidal antiinflammatory drugs, and therapeutic exercise program before joining the study). No calcium supplementation was given during the study period.

\section{Statistical Analyses and Sample Size for the Study}

Statistical analyses were performed using SPSS Version 23 (IBM Corporation, Armonk, NY). As per the previous studies, we calculated that to estimate an effect size of 0.56 , we needed at least 20 patients in each group at beta of $90 \%$ and alpha error of $0.05(10,12,14)$. Mean and medians were calculated for all quantitative variables and measures of dispersion, standard deviation, or standard error were calculated. Normality of data were reviewed using the Kolmogorov-Smirnov test. For normally distributed data, means of 2 groups were compared using the $t$ test. For skewed data, the Mann-Whitney $U$ test was applied. Qualitative or categorical variables were described as frequencies and proportions. Proportions were compared using the chi-square test. For time-dependent repeated variables such as VAS and MODQ scores, repeated measures analysis of variance (ANOVA) was applied. To estimate mean change in VAS and MODQ scores, one-way ANOVA was used followed by Bonferroni correction. Serial values of serum calcium, serum vitamin D3, serum osteocalcin, and DEXA scan were compared using the paired $t$ test.

\section{RESULTS}

Figure 1 shows the CONSORT of patients. Demographic data, baseline VAS and MODQ scores were comparable between the 3 groups (Table 1). Three hundred and fifty-one patients were screened, and 65 patients were included in the study. There was a loss to follow-up in 4 patients. Data were analyzed for 61 patients: 38 patients had single disc herniation, and 23 patients had disc herniation at 2 levels. The number of patients with one or 2 disc herniation were equally distributed in the 3 groups.

\section{Primary Outcome}

\section{Effectiveness of Intervention}

Effectiveness of intervention defined as $50 \%$ re- duction in VAS scores at 6 months showed a statistically significant difference among the 3 groups $(P=0.024$; chi-square test). On subgroup analysis, group PIL (16 of 20 ) and group TF (15 of 20) were significantly more effective than group MIL (12 of 21) $(P=0.016$ and $P$ $=0.038$, respectively; chi-square test). However, groups PIL and TF were comparable in effectiveness of intervention ( $P=0.50$; chi-square test) (Fig. 2).

Mean number of injections administered to achieve $50 \%$ reduction in pain scores in the 3 groups were similar (group MIL $2.33 \pm 0.65$, group PIL $2.10 \pm 0.64$, group TF $2.05 \pm 0.68, P=0.347$; one-way ANOVA). Percentage of patients who received only one ESI for effective pain relief were 2 of $21(9.5 \%)$ in group MIL, 3 of $20(15 \%)$ in group PIL, and 5 of $20(25 \%)$ in group TF. Additionally, 10 of $21(47.6 \%)$ patients in group MIL, 12 of $20(60 \%)$ in group PIL, and 10 of $20(50 \%)$ in group TF required 2 ESIs for effective pain relief. Percentages of patients receiving 3 ESIs for effective pain relief were 9 of 21 $(42.9 \%)$ in group MIL, 5 of $20(25 \%)$ in group PIL, and 5 of $20(25 \%)$ in group TF (Fig. 3).

\section{Secondary Outcome}

\section{VAS Score}

Repeated measures ANOVA showed significant difference in serial VAS scores between the 3 groups over the follow-up time $(P=0.00)$. We observed that all modalities were effective in each of the groups, as most of the patients reported a significant decrease in VAS score from baseline at all time intervals, that is at 15 days, 1-month, 3-month, and 6-month follow-up (VAS time interaction). VAS scores at 3- and 6-month follow-up showed statistically significant difference in pain scores among the 3 groups (group MIL $3.81 \pm 1.25$, group PIL $2.7 \pm 0.86$, group TF $3.05 \pm 0.75, P=0.002$ at 3 months, and group MIL $3.86 \pm 1.01$, group PIL $2.35 \pm 0.74$, group TF $2.45 \pm 0.51, P=0.000$ at 6 months; one-way ANOVA). Further, patients receiving ESI in group PIL and group TF showed significantly lower VAS scores than group MIL $(P=0.02, P=0.50$ at 3 months, and $P=0.00, P=0.02$ at 6 months, respectively; Bonferroni correction). However, group PIL and group TF did not significantly differ in VAS score $(P=0.799$ at 3 months, and $P=0.972$ at 6 months; Bonferroni correction) (Fig. 4).

\section{MODQ}

Repeated measures ANOVA revealed significant MODQ time interaction within the 3 groups, and MODQ group interaction after epidural injection. Statistically 


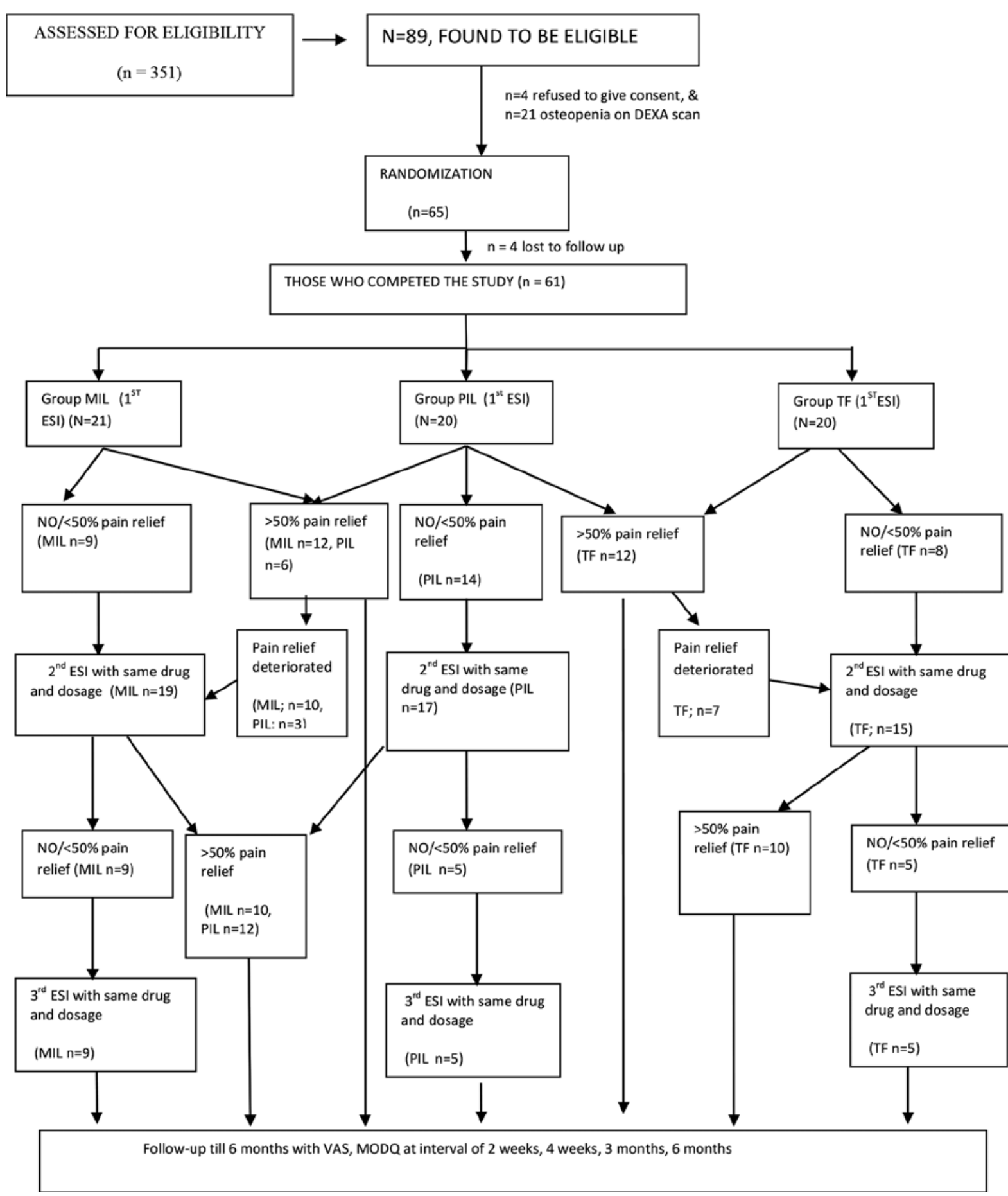

Fig. 1. CONSORT statement. 
Table 1. Demographic data, baseline VAS and MODQ scores.

\begin{tabular}{|l|c|c|c|c||}
\hline & $\begin{array}{c}\text { Group MIL } \\
(\mathbf{n}=\mathbf{2 1})\end{array}$ & $\begin{array}{c}\text { Group PIL } \\
(\mathbf{n}=\mathbf{2 0})\end{array}$ & $\begin{array}{c}\text { Group TF } \\
(\mathbf{n}=\mathbf{2 0})\end{array}$ & P Value \\
\hline Age (years)* & $42.71( \pm 7.47)$ & $41.15( \pm 7.38)$ & $37.65( \pm 6.72)$ & 0.08 \\
\hline $\begin{array}{l}\text { Gender } \\
\text { Male } \\
\text { Female }\end{array}$ & $\begin{array}{c}12(57.1 \%) \\
9(42.9 \%)\end{array}$ & $\begin{array}{c}7(33.3 \%) \\
14(66.7 \%)\end{array}$ & $\begin{array}{c}11(47 . \%) \\
12(52.2 \%)\end{array}$ & 0.158 \\
\hline Height $(\mathrm{cm})^{*}$ & $162.62( \pm 7.76)$ & $159.05( \pm 7.59)$ & $164.45( \pm 9.66)$ & 0.126 \\
\hline Weight $(\mathrm{kg})^{*}$ & $67.24( \pm 8.780)$ & $63.35( \pm 8.00)$ & $66.95( \pm 11.16)$ & 0.349 \\
\hline $\begin{array}{l}\text { Body mass index } \\
\left(\mathrm{kg} / \mathrm{m}^{2}\right)^{*}\end{array}$ & $25.46( \pm 3.262)$ & $25.42( \pm 2.08)$ & $24.75( \pm 3.60)$ & 0.708 \\
\hline Baseline VAS* & $7.90( \pm 0.625)$ & $7.88( \pm 0.972)$ & $7.80( \pm 0.951)$ & 0.923 \\
\hline Baseline MODQ & $60.33( \pm 2.921)$ & $61.20( \pm 4.275)$ & $60.75( \pm 3.582)$ & 0.747 \\
\hline $\begin{array}{l}\text { Number of disc } \\
\text { herniations } \\
1\end{array}$ & $13(61.9 \%)$ & $14(70 \%)$ & $11(55 \%)$ & \\
2 & $8(38.1 \%)$ & $6(30 \%)$ & $9(45 \%)$ & \\
\hline
\end{tabular}

* - data expressed as mean \pm SD

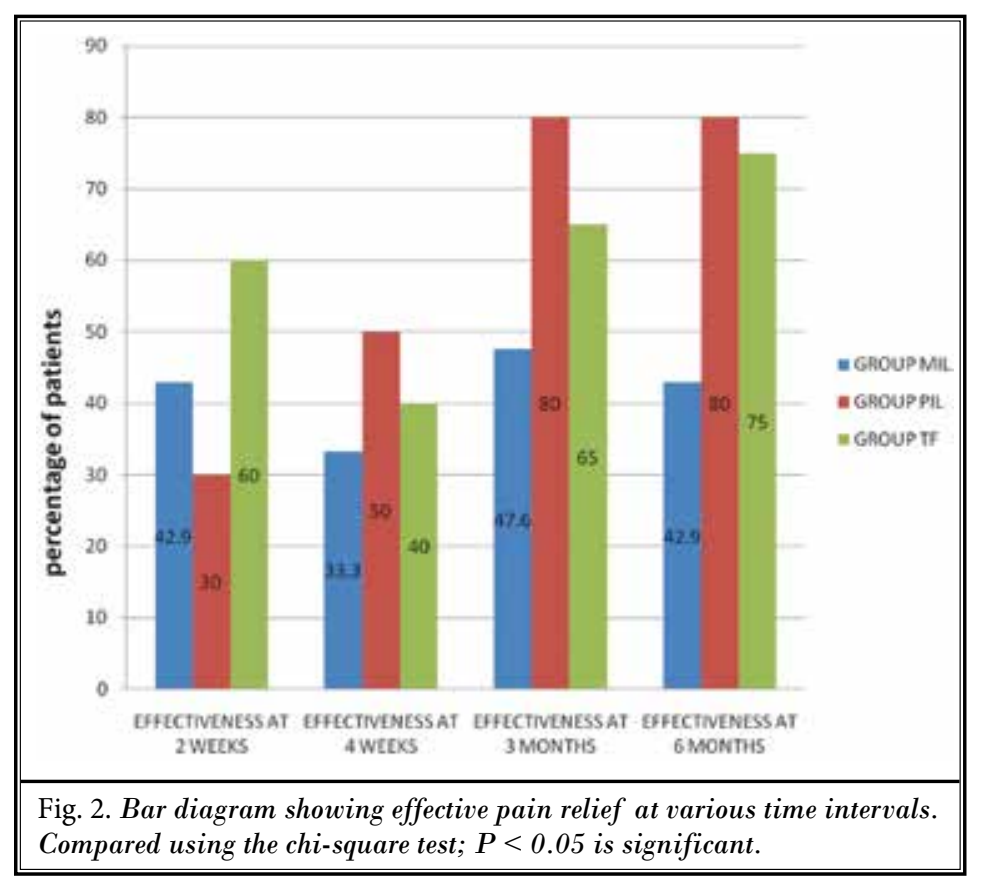

significant difference in MODQ scores were noted among the 3 groups at 3 months (group MIL $41.76 \pm 8.64$, group PIL $30.65 \pm 5.76$, group TF $30.75 \pm 5.76 ; P=0.000$ ) and 6 months (group MIL $37.81 \pm$ 7.21, group PIL $23.70 \pm 5.13$, group TF $24.15 \pm 3.18 ; P=0.000)$ using one-way ANOVA (Fig. 5).

\section{Contrast Medium Spread Pattern}

A total of 132 ESIs were administered during various time intervals described in the protocol in our study (group MIL 49, group PIL 42, and group TF 41).
There was significant difference between percentage of patients demonstrating unilateral or bilateral spread among the 3 groups $(P<0.00$; chi-square test). On individual group comparison, TF had a greater number of unilateral contrast medium spread (41 of $41,100 \%$ ), followed by group PIL (28 of 42, 66.7\%). A significant difference between percentages of patients having unilateral or bilateral spread was observed between groups (MIL and PIL, $P<0.000$; groups PIL and TF, $P$ $<0.000$; and groups MIL and TF, $P<0.000$; chi-square test). Anterior spread was seen in only $51 \%$ of ESIs performed in the MIL group as compared with $85.7 \%$ in the PIL group ( $P<0.00$; chi-square test). Further anterior spread was seen in $82.9 \%$ of ESIs performed in the TF group ( $P=0.002$; chisquare test) when compared with the MIL group. However, there was no significant difference observed between percentages of anterior spread of contrast medium between group PIL and TF $(P=0.727$; chisquare test).

Perineural spread was significantly different among the 3 groups $(P=$ 0.00; chi-square test). Group TF (85.4\%) showed a statistically significant better spread in comparison to groups PIL (57.1\%; $P=0.005)$ and MIL (16.3\%; $P=$ $0.000)$. Further, better perineural spread was observed in group PIL than group MIL $(P=0.000)$.

The average number of vertebral segments of cephalic spread of contrast medium in group MIL was $2.88 \pm 1.092$, group PIL was $3.21 \pm 1.001$, and in group TF was $3.15 \pm 0.937$. There was no statistically significant difference between means values of cephalic spread between the 3 groups ( $P=0.247$, one-way ANOVA).

Fluoroscopy exposure time was significantly different in the 3 study groups $(P=0.00$; one-way ANOVA). On betweengroup comparisons, ESIs in group TF took more fluoroscopy time $(13.34 \pm 1.353 \mathrm{sec}-$ onds), followed by group PIL $(9.10 \pm 0.878$ seconds). There was significant difference in fluoroscopy time between group MIL 


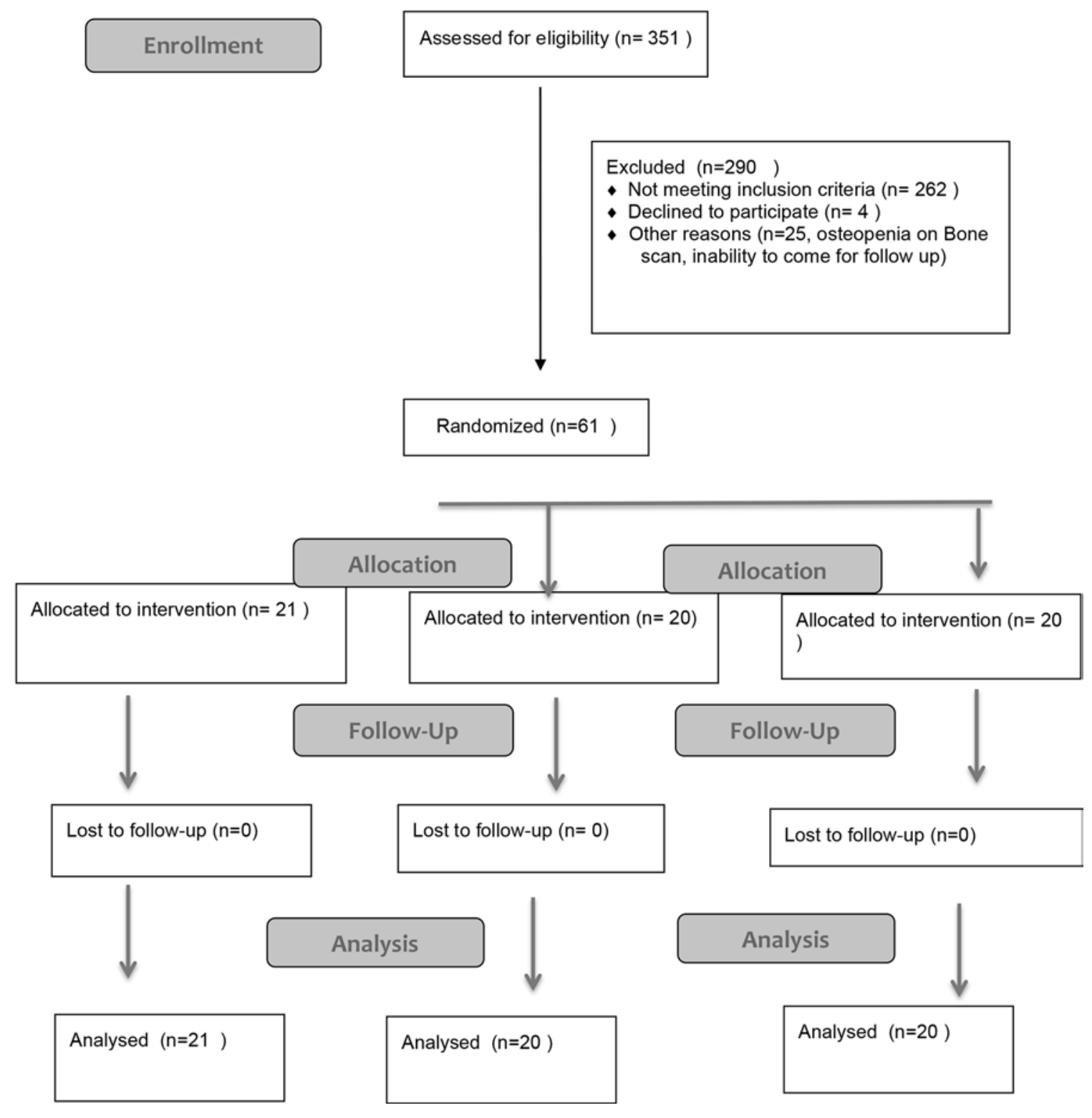

Fig. 3. Flow of the patients.

and PIL, group MIL and TF, and in group PIL and TF $(P=$ $0.029, P=0.000, P=0.00$, respectively; post hoc analysis using the Bonferroni test).

There was no statistically significant difference in mean serum osteocalcin levels, serum vitamin D3 levels, and mean DEXA hip and spine $t$ and $z$ scores among the 3 groups at baseline and at 3 months (Table 2).

\section{Discussion}

The present study was designed to compare the therapeutic effectiveness of 3 routes for administering ESI in patients with unilateral radicular LBP not responding to conservative treatment. Because the use of neuraxial steroid may induce osteoporosis secondary to increased bone resorption and decreased 


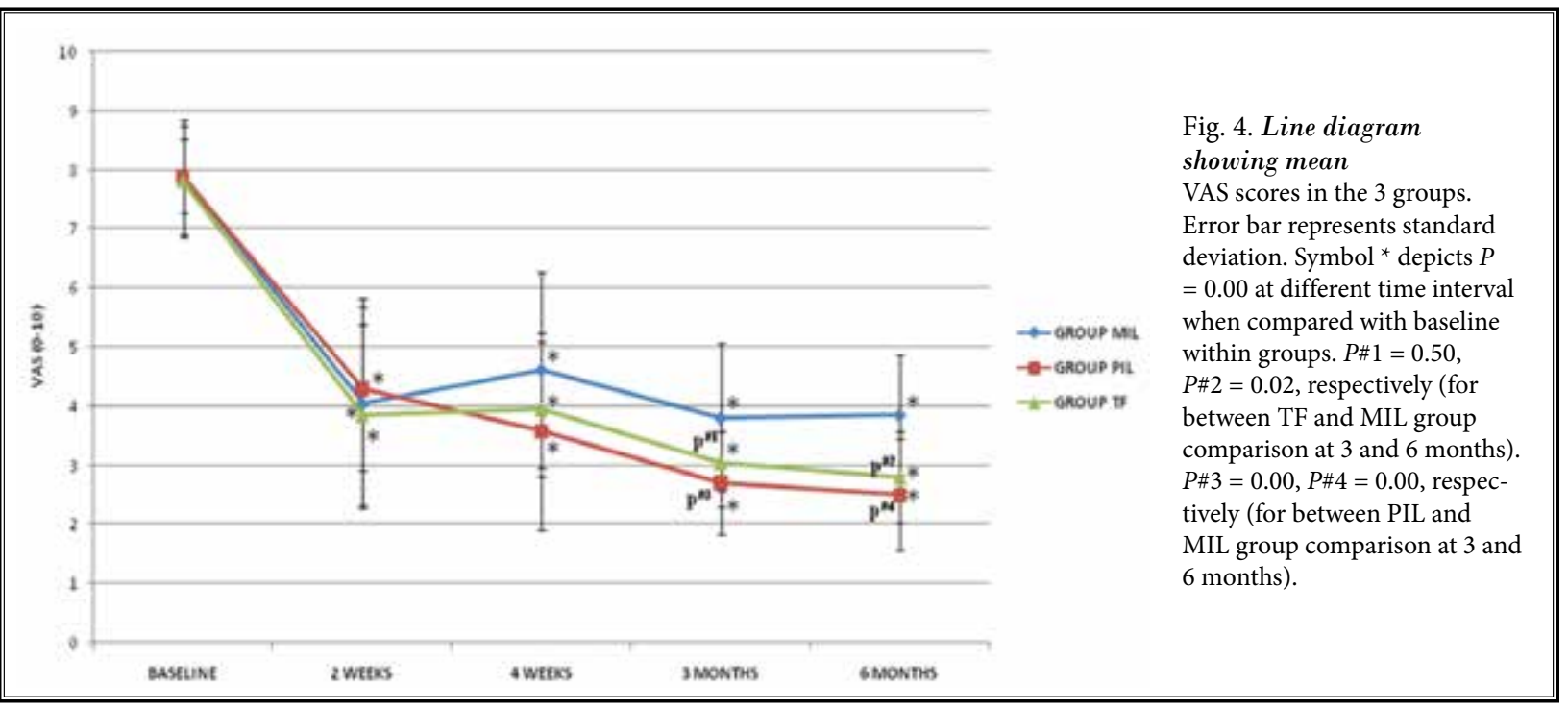

Fig. 5. Line diagram showing mean $M O D Q$ scores in the 3 groups.

Error bar represents standard deviation. Symbol $\left({ }^{*}\right)$ depicts $P=0.00$ at different time interval when compared with baseline within groups. $P \# 1=0.00, P \# 2=0.00$, respectively (for between TF and MIL group comparison at 3 and 6 months). $P \# 3$ $=0.00, P \# 4=0.00$, respectively (for between PIL and MIL group comparison at 3 and 6 months).

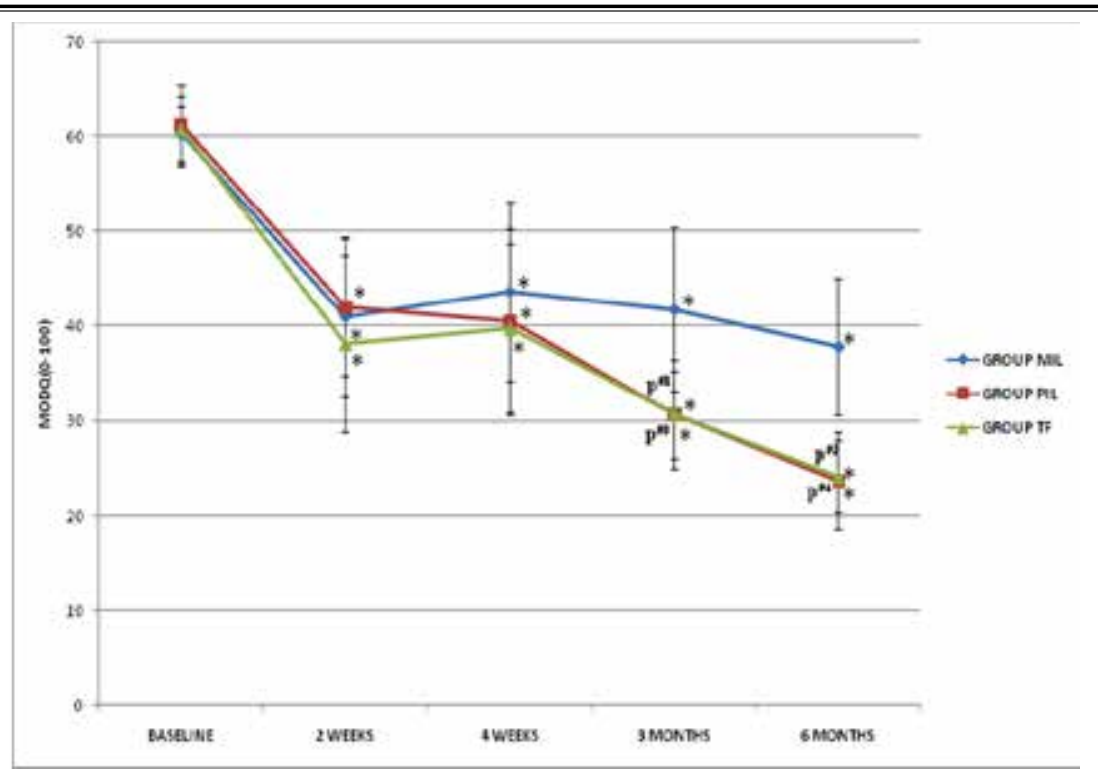

bone formation, effect of epidural steroid on BMD using DEXA scan was evaluated $(21,22)$. We found that $80 \%$ of patients reported effective pain relief in the PIL group, followed by $75 \%$ in the TF group, and only $43 \%$ in the MIL group. There was a significant reduction in VAS and MODQ scores in all 3 groups as compared with baseline. However, decrease in VAS and MODQ scores were greater in group TF and PIL as compared to group MIL at 3 and 6 months. We did not find any difference in number of injections required in the 3 groups for effective pain relief. The spread of contrast medium on fluoroscopy showed better anterior spread in patients receiving ESI using PIL and TF approach as compared with MIL. Mean serum osteocalcin levels, serum vitamin D3 levels, and mean DEXA hip and spine $t$ and $z$ scores did not differ significantly from the baseline.

Ackerman and Ahmad (9) conducted a randomized study comparing 3 approaches: TF, IL, and caudal ESI in patients with disc herniation. The authors reported 
better pain relief in patients receiving ESI through the TF approach than the IL and caudal approach. This was attributed to placement of drug closer to the site of pathology. In a prospective case-control study, Schaufele et al (17) compared IL and TF approaches with the primary objective of observing improvement in pain. They also reported that TF ESIs were superior to IL in terms of both pain improvement and long-term surgical intervention. In both studies, authors reported better outcomes using the TF approach. However, recent research has concluded that the IL approach is not inferior to the TF approach. Gharibo et al (21) compared the TF and IL approach in patients with LBP due to disc herniations, and reported significant improvement in pain and function with both approaches. The dose of drug used in the 2 groups was different ( $40 \mathrm{mg}$ triamcinolone in the TF group, and $80 \mathrm{mg}$ of triamcinolone in the IL group). Second, patients were followed for a short duration of 10 to 16 days only. Rados et al (22) compared the TF and IL approaches in patients with chronic lumbar radiculopathy. The authors demonstrated a significant decrease in VAS scores with both approaches. Again, authors used a higher dose of methylprednisolone in group IL (80 mg) as compared with group TF $(40 \mathrm{mg})$. A total of $53 \%$ of ESIs were successful in the IL group, and $63 \%$ successful in the TF group. Most of the studies using $80 \mathrm{mg}$ of methylprednisolone in the TF group reported a success rate of $70 \%$ to $80 \%(9,11)$. It appears that Rados et al (22) probably did not administer equivalent doses in either approaches, and therefore found IL to be as effective as TF.

Although more efficacious, incidence of severe complications in the TF approach are higher. This may be owing to embolization of the artery of Adamkiewicz accompanying the nerve root (15). Some $11.2 \%$ of intravascular injections have been reported in the TF approach as compared with $1.9 \%$ with IL ESIs $(23,24)$. The PIL approach may provide a suitable alternative to the TF approach in which the drug is deposited close to pathology similar to the TF approach, but at the same time avoids complications associated with TF $(10,12-13)$. Furman et al (25) conducted a pilot study to evaluate the effective pain relief using the PIL approach in patients with lumbar radiculopathy and showed significant improvement in pain scores at 3 months. Because it was a single arm pilot study, further studies addressing this issue are required (25).

Candido et al (10) conducted a randomized study in which IL injection was performed in the lateral most part of the epidural space (i.e., PIL) in one group and
Table 2. Data comparing biochemical investigations at 3 months with baseline.

\begin{tabular}{|c|c|c|c|}
\hline & & $\begin{array}{c}\text { Mean } \pm \text { Standard } \\
\text { Deviation }\end{array}$ & $P$ Value \\
\hline \multirow{2}{*}{ Osteocalcin } & Baseline & $9.080 \pm 3.753$ & \multirow{2}{*}{0.077} \\
\hline & 3 months & $11.877 \pm 12.106$ & \\
\hline \multirow{2}{*}{ Vitamin D3 } & Baseline & $31.059 \pm 0.945$ & \multirow{2}{*}{0.437} \\
\hline & 3 months & $31.5843 \pm 0.889$ & \\
\hline \multirow{2}{*}{ Serum calcium } & Baseline & $9.384 \pm 0.447$ & \multirow{2}{*}{0.279} \\
\hline & 3 months & $9.430 \pm 0.344$ & \\
\hline \multirow{2}{*}{$\begin{array}{l}\text { DEXA scan } \\
\text { hip T }\end{array}$} & Baseline & $-0.266 \pm 0.69$ & \multirow{2}{*}{0.085} \\
\hline & 3 months & $-0.310 \pm 0.67$ & \\
\hline \multirow{2}{*}{$\begin{array}{l}\text { DEXA scan } \\
\text { hip Z }\end{array}$} & Baseline & $-0.185 \pm 0.68$ & \multirow{2}{*}{0.081} \\
\hline & 3 months & $-0.220 \pm 0.68$ & \\
\hline \multirow{2}{*}{$\begin{array}{l}\text { DEXA scan } \\
\text { spine } \mathrm{T}\end{array}$} & Baseline & $-0.46 \pm 0.85$ & \multirow{2}{*}{0.086} \\
\hline & 3 months & $-0.472 \pm 0.83$ & \\
\hline \multirow{2}{*}{$\begin{array}{l}\text { DEXA scan } \\
\text { spine } Z\end{array}$} & Baseline & $-0.408 \pm 0.83$ & \multirow{2}{*}{0.601} \\
\hline & 3 months & $-0.39 \pm 0.82$ & \\
\hline
\end{tabular}

Data expressed as mean \pm standard deviation, analyzed using paired $t$ test. $P<0.05$ was significant.

TF was performed in the second group. The authors reported comparable VAS scores at all time intervals, that is 2 weeks, 4 weeks, 1 month, 3 months, and 6 months using either approach. Similarly studies conducted by Ghai et al (12) and Hashemi et al (13) also reported comparable pain scores during follow-up using PIL and TF approaches.

Evidence comparing pain relief using MIL with PIL was reported in a single study by Ghai et al (11). The authors found a higher incidence of effective pain relief at 6 months in the PIL group $(68.4 \%)$ as compared with the MIL group (16.7\%). However, the success rate of $16.7 \%$ found in the MIL group in the study was lower than that reported in several studies $(20,21)$. We compared the therapeutic effectiveness of the 3 routes for administering ESI in patients with unilateral radicular LBP not responding to conservative treatment and found that the PIL and TF techniques were comparable in effectiveness of intervention.

Lee et al (26) conducted a meta-analysis to investigate efficacy of TF with IL epidural and reported significantly better short-term pain relief. However, quality of evidence for these results were low indicating need of more robust RCTs. Further, authors did not perform a subgroup analysis between MIL and PIL techniques of ESI (26).

Ventral spread of contrast medium on fluoroscopy signifying greater drug deposition was observed in 
most of the patients in the PIL group (85.7\%) and in the TF group (82.9\%). The previous data studying contrast medium spread has also reported better ventral spread in PIL and TF approaches similar to our study $(10,12)$.

The use of steroid is associated with many adverse effects (27). Glucocorticoid induced osteoporosis is one of the common adverse effects and varies with preparations, duration, dose, and route of administration. Although enough studies have shown decrease in BMD and decrease in bone quality with use of oral or inhalational steroids, limited studies have evaluated the effect of epidural steroids on BMD.

Manchikanti et al (18) conducted a prospective study to evaluate the effect of neuraxial steroid on BMD. The authors demonstrated no significant change in BMD at 3 months, 6 month, and one year in the group receiving neuraxial steroids as compared with baseline (18). This may be because of the very low doses of steroids used in the study. In the study, betamethasone of 3 to $6 \mathrm{mg}$ (equivalent to 20 to 40 $\mathrm{mg}$ of methylprednisolone) was used in the caudal approach, and 1.5 to $3 \mathrm{mg}$ betamethasone (equivalent to 10 to $20 \mathrm{mg}$ of methylprednisolone) for the TF approach. As doses routinely used in epidural space are greater than these, results of the study need to be interpreted with caution. Al-Shoha et al (19) evaluated the effect of ESI on BMD in 1,000 patients. The authors reported a significant mean difference in BMD of the hip at 6 months. Study population consisted of postmenopausal women who were most vulnerable to bone loss, a very well-known fact. Another retrospective analysis reported that ESI caused BMD changes in postmenopausal women not taking antiosteoprotic medications (28). However, our study found no significant difference in serum calcium, serum osteocalcin, serum vitamin D3, or DEXA hip and spine score values at 3 months from baseline values.

We did not observe any dural puncture or any spread of contrast medium in subarachnoid space, subdural space, and intradiscal space. No patient developed skin lesions or paresthesia. Postdural puncture headache occurred in one patient only, and responded to conservative treatment.

Even with a midline approach, in the majority of the time, the epidural needle ends up being parasagittal or at least paramedian while performing the procedure. To prevent this, different entry points were targeted for the midline and parasagittal approach. The needle was introduced at the midpoint between 2 spinous processes at the effected level in the MIL approach. Final position of needle in-between the 2 spinous processes was confirmed with fluoroscopy before giving contrast medium. However, in the PIL approach, the needle was introduced into the most lateral epidural space of the effected side.

Our decision to use $4 \mathrm{~mL}$ was based on the available literature on ESI in the last 10 years in which maximum studies have used a volume ranging from 3 to 10 $\mathrm{mL}(9,15,25)$. Also, Makkar et al $(29)$ found no increase in effectiveness of ESI volume of injectate that was increased from 4 to $8 \mathrm{~mL}$.

All TF procedures were performed under real-time fluoroscopy. Vascular puncture was observed in 2 patients in the TF group and required needle relocation. Intravascular injection can also occur in the epidural space secondary to needle placement in the vertebral venous plexus. As posterior internal vertebral venous plexus attenuates in the midline, incidence of intravascular uptake reported in literature during translaminar injection is as low as $2 \%$ (24). It is therefore possible to have a higher incidence of intravascular injection with parasagittal epidural injection secondary to placement of the needle in the lateral epidural vein. We did not observe any vascular injection in group PIL.

Several case reports have documented potential complications such as paraplegia secondary to spinal cord infarction associated with the use of the TF technique $(14,23)$. Incidents have been associated with both safe triangle approach as well as use of particulate steroid. Particulate steroids can cause occlusion of the segmental artery or vertebral artery resulting in cord ischemia $(30,31)$. However, available literature suggests that particulate steroids offer a slightly better VAS score as compared with nonparticulate steroids, and clinicians need to weigh the advantage offered by this difference with the complications reported in the literature with use of particulate steroids (32). To address this issue, a working group constituted under safe use initiative of the US Food and Drug Administration (FDA) issued a warning that injection of corticosteroids into the epidural space of the spine may result in rare, life-threatening complications (33). Manchikanti et al (34) criticized this warning, stating the FDA failed to discuss simple measures to prevent neurologic complications such as initiation of alternate techniques to classic and traditional teachings, avoidance of particulate steroids, use of a blunt needle, and differentiation between different techniques of epidural injection. We used extension tubing to reduce the risk of the needle getting dislodged at the time of change of syringes. All 
TF procedures in our study were performed under realtime fluoroscopy.

We used the subpedicular "safe triangle" approach. However, the term "safe" is in reference to the location of the neural but not the vasculature structures. In light of anatomic and radiologic evidence that radicular arteries dwell in the superior part of the foramina, use of alternate techniques have been suggested in literature (35). Many practitioners have proposed adopting the retrodiscal (infraneural) approach with the needle tip positioned into Kambin's triangle $(36,37)$. Although final position of the needle in this technique might result in less likelihood of radiculomedullary intraarterial cannulation, there may be other risks associated with this needle tip placement. The needle tip may unintentionally be placed too far ventrally and enter the intervertebral disc (38), and risk of subarachnoid or subdural injection may be higher because theoretically, the axillary pouch of the nerve-root sleeve may encroach into Kambin's triangle. In a retrospective review published by Levi et al (39), the authors reported inadvertent intradiscal injections in $4.7 \%$ of patients, and intrathecal injections in $3.1 \%$ of patients with use of infraneural/retrodiscal technique. Beyond safety issues in choosing a TF approach, many physicians believe one approach is superior to another for a variety of reasons. At this point in time, it remains unclear if the infraneural or traditional subpedicular technique is superior in effectiveness. Few small trials have investigated this issue without demonstrating any significant clinical difference between the 2 approaches $(38,40)$.

Interventional techniques are one of the commonly used modalities in treating chronic pain, with increasing use and debate to effectiveness. There continues to be a lack of agreement between proponents and opponents on lack of efficacy of certain interventional techniques along with increasing burden of cost (41-45).

RCTs are the gold standard in the evidence-based evaluation of efficacy of intervention modalities/ treatment, and are different from other study designs because they are performed under very rigorous conditions. An RCT uses randomization, control group, and double-blind design to minimize bias. There are 2 distinct ways to show efficacy of a particular intervention in an RCT comparison with a (1) placebo control or (2) an active control. RCT with a control assures the investigator that observed treatment effects can be attributed to the intervention, rather than to external factors. No information external to the trial is needed to support the conclusion of effectiveness. However, a study with active control shows that the new therapy is equivalent to or not worse by some defined amount than a known effective treatment (46). Placebo controlled trials are inappropriate in the field of chronic pain, as a delay in treatment increases the disability of the patient and increases the financial burden on society. Active control equivalence trials can be used more appropriately in many systematic reviews and evidence synthesis over single armed trials (47).

There are a few limitations of our study. First, there was no placebo group/control group. This was justifiable because it would be unethical to have a placebo group in which patients are denied pain relief. Second, DEXA scan for BMD was not recorded at 1 and 6 months. Third, we did not study various factors that might have influenced the outcome of our study (48). Our study is limited by a small sample size. For the purpose of the study, only patients with the earlier mentioned fairly strict inclusion/exclusion criteria were enrolled. This eliminates a large proportion of patients typically seen by an Interventional Pain Physician. ESIs are commonly performed for radicular symptoms in patients with multilevel disc herniations, previous blind ESI, and for diagnostic reasons in cases of an unclear diagnosis. It was our goal to limit the study to a welldefined patient population to increase the validity of our results. Finally, we followed our patients for only 6 months. Longer follow-up were desirable to know the long-term effectiveness of the procedure.

\section{Conclusions}

The PIL approach is equivalent to the TF approach in terms of effective pain relief and improvement in disability, and superior to the MIL approach in a patient with unilateral lumbar radiculopathy. The PIL approach can be considered as a suitable alternative to the TF approach for administering ESI as both PIL and TF approaches are equally effective, and the PIL approach is associated with fewer complications and technically easy to perform. Low-dose neuraxial steroids are safe in patients with lumbar radiculopathy. This study showed no deleterious effect on BMD. 


\section{References}

1. Manchikanti L. Transforaminal lumbar epidural steroid injection. Pain Physician 2000; 3:374-398.

2. Murphy RW. Nerve roots and spinal nerves in degenerative disc disease. Clin Ortho 1977; 129;46-6o.

3. McCarron RF, Wimpee MW, Hudkins $P G$, Laros GS. The inflammatory effect of nucleus pulposus. A possible element in pathogenesis of low back pain. Spine 1987; 12:760-764.

4. Carreon LY, Glassman SD, Howard J. Fusion and nonsurgical treatment for symptomatic lumbar degenerative disease: A systematic review of Oswestry Disability Index and MOS Short Form36 outcomes. Spine J 2008; 8:747-755.

5. Chou R, Atlas SJ, Stanos SP, Rosenquist RW. Nonsurgical interventional therapies for low back pain: A review of the evidence for an American Pain Society clinical practice guideline. Spine 2009; 34:1078-1093.

6. Saal JS, Franson RC, Dobrow R, Saal JA, White AH, Goldthwaite N. High levels of inflammatory phospholipase A2 activity in lumbar disc herniations. Spine 1990; 15:674-678.

7. O'Donnell JL, O'Donnell AL. Prostaglandin E2 content in herniated lumbar disc disease. Spine 1996; 21:1653-1655.

8. Molloy RE, Benzon HT. Interlaminar epidural steroid injections for lumbosacral radiculopathy. In: Benzon HT, Raja SN, Molloy RE, Liu SS, Fishman SM (eds). Essentials of Pain Medicine and Regional Anesthesia. 2nd ed. Philadelphia, Elsevier Churchill Livingstone, 2005: pp. 331-332.

9. Ackerman WE 3rd, Ahmad M. The efficacy of lumbar epidural steroid injections in patients with lumbar disc herniations. Anesth Analg 2007; 104:1217-1222.

10. Candido KD, Raghavendra MS, Chinthagada M, Badiee S, Trepashko DW. A prospective evaluation of iodinated contrast flow patterns with fluoroscopically guided lumbar epidural steroid injections: The lateral parasagittal interlaminar epidural approach versus the transforaminal epidural approach. Anesth Analg 2008; 106:638-644.

11. Ghai B, Vadaje KS, Wig J, Dhillon MS. Lateral parasagittal versus midline interlaminar lumbar epidural steroid injection for management of low backpain with lumbosacral radicular pain: $A$ double-blind, randomized study. Anesth Analg 2013; 117:219-227.
12. Ghai B, Bansal D, Kay JP. Transforaminal versus parasagittal interlaminar epidural steroid injection in low back pain with radicular pain: A randomized, doubleblind, active-control trial. Pain Physician 2014; 17:277-290.

13. Hashemi SM, Aryani MR, Momenzadeh S. Comparison of transforaminal and parasagittal epidural steroid injections in patients with radicular low back pain. Anesth Pain Med 2015; 5:e26652.

14. Huntoon MA, Martin DP. Paralysis after transforaminal epidural injection and previous spine surgery. A case report. Reg Anesth Pain Med 2004; 29:494-495.

15. Furman MB, O'Brien EM, Zgleszewski TM. Incidence of intravascular penetration in transforaminal lumbosacral epidural steroid injections. Spine 2000; 25:2628-2632.

16. Chang Chien GC, Candido KD, Knezevic NN. Digital subtraction angiography does not reliably prevent paraplegia associated with lumbar transforaminal epidural steroid injection. Pain Physician 2012; 15:515-523.

17. Schaufele MK, Hatch L, Jones W. Interlaminar versus transforaminal epidural injections for the treatment of symptomatic lumbar intervertebral disc herniations. Pain Physician 2006; 9:361-366.

18. Manchikanti L, Pampati V, Beyer C. The effect of neuraxial steroids on weight and bone mass density: A prospective evaluation. Pain Physician 2000; 3:357-366.

19. Al-Shoha A, Rao DS, Schilling J, Peterson E, Mandel S. Effect of epidural steroid injection on bone mineral density and markers of bone turnover in postmenopausal women. Spine (Phila Pa 1976) 2012; 37:E1567-E1571.

20. Bicket MC, Gupta A, Brown $\mathrm{CH}$, Cohen SP. Epidural injections for spinal pain: A systematic review and meta-analysis evaluating the "control" injections in randomized controlled trials. Anesthesiology 2013; 119:907-931.

21. Gharibo CG, Varlotta GP, Rhame EE, Liu EC, Bendo JA, Perloff MD. Interlaminar versus transforaminal epidural steroids for the treatment of subacute lumbar radicular pain: A randomized, blinded, prospective outcome study. Pain Physician 2011; 14:499-511.

22. Rados I, Sakic K, Fingler M, Kapural L. Efficacy of interlaminar vs. transforaminal epidural steroid injection for the treatment of chronic unilateral radicular pain: Prospective, randomized study.
Pain Med 2011; 12:1316-1321.

23. Houten JK, Errico TJ. Paraplegia after lumbosacral nerve root block: Report of three cases. Spine ] 2002; 2:70-75.

24. Sullivan WJ, Willick SE, Chira-Adisai W. Incidence of intravascular uptake in lumbar spinal injection procedures. Spine 2000; 25:481-486.

25. Furman MB, Kothari G, Parikh T, Anderson JG, Khawaja A. Efficacy of fluoroscopically guided, contrast-enhanced lumbosacral interlaminar epidural steroid injections: A pilot study. Pain Med 2010; 11:1328-1334.

26. Lee JH, Shin KS, Park S), et al. Comparison of clinical efficacy between transforaminal and interlaminar epidural injections in lumbosacral disc herniation: A systematic review and meta-analysis. Pain Physician 2018; 21:433-448.

27. Abdul AJ, Ghai B, Bansal D, Sachdeva N, Bhansali A, Dhatt SS. Hypothalamic pituitary adrenocortical axis suppression following a single epidural injection of methylprednisolone acetate. Pain Physician 2017; 20:E991-E1001.

28. Kim $\mathrm{YU}$, Karm $\mathrm{MH}$, Cheong $\mathrm{Y}$, et al. Effect of epidural steroid injection on bone mineral density in postmenopausal women according to antiosteoporotic medication use. Pain Physician 2016; 19:389-396.

29. Makkar JK, Kumar B, Jain K, Dhutt SS, Batra YK, Singh PM. Effect of different volumes on pain relief in patient receiving fluoroscopic guided interlaminar lumbar epidural steroid injection. Pain Physician 2018; 21:243-250.

30. Rozin L, Rozin R, Koehler SA, et al. Death from transforaminal epidural steroid nerve root block $\left(\mathrm{C}_{7}\right)$ due to perforation of the left vertebral artery. Am J Forensic Med Pathol 2003; 24:351-355.

31. Tiso RL, Cutler T, Catania JA, Whalen K. Adverse central nervous system sequelae after selective transforaminal block: The role of corticosteroids. Spine J 2004; 4:468-474.

32. Makkar JK, Singh PM, Jain D, Goudra B. Particulate vs non-particulate steroids for transforaminal epidural steroid injections: Systematic review and metaanalysis of the current literature. Pain Physician 2016; 19:327-340.

33. Rathmell JP, Benzon HT, Dreyfuss P, et al. Safeguards to prevent neurologic complications after epidural steroid injections: Consensus opinions from a multidisciplinary working group and 
national organizations. Anesthesiology 2015; 122:974-984.

34. Manchikanti L, Falco FJ. Safeguards to prevent neurologic complications after epidural steroid injections: Analysis of evidence and lack of applicability of controversial policies. Pain Physician 2015; 18:E129-E138.

35. Atluri S, Glaser SE, Shah RV, Sudarshan G. Needle position analysis in cases of paralysis from transforaminal epidurals: Consider alternative approaches to traditional techniques. Pain Physician 2013; 16:321-334.

36. Chang J. Lumbar transforaminal epidural steroid injections through a reverse safe triangle approach-technical consideration. Pain Med 2011; 12:1439-1440.

37. Glaser SE, Shah RV. Root cause analysis of paraplegia following transforaminal epidural steroid injections: The 'unsafe' triangle. Pain Physician 2010; 13:237-244.

38. Kim C, Moon CJ, Choi HE, Park Y. Retrodiscal approach of lumbar epidural block. Ann Rehab Med 2011; 35:418-426.

39. Levi D, Horn S, Corcoran S. The incidence of intradiscal, intrathecal, and intravascular flow during the performance of retrodiscal (infraneural) approach for lumbar transforaminal epidural steroid injections. Pain Med 2016; 17:1416-1422.

40. Park KD, Lee J, Jee H, Park Y. Kambin triangle versus the supraneural approach for the treatment of lumbar radicular pain. AmJ Phys Med Rehabil 2012; 91:1039-1050.

41. Manchikanti L, Pampati V, Benyamin RM, Hirsch JA. Cost utility analysis of lumbar interlaminar epidural injections in the treatment of lumbar disc herniation, central spinal stenosis, and axial or discogenic low back pain. Pain Physician 2017; 20:219-228.

42. Manchikanti L, Hirsch JA, Pampati V, Boswell MV. Utilization of facet joint and sacroiliac joint interventions in Medicare population from 2000 to 2014 : Explosive growth continues! Curr Pain Headache Rep 2016; 20:58.

43. Manchikanti L, Soin A, Mann DP, et al. Utilization patterns of facet joint interventions in managing spinal pain: $A$ retrospective cohort study in the US feefor-service Medicare population. Curr Pain Headache Rep 2019; 23:73.

44. Manchikanti L, Soin A, Mann DP, Bak- shi S, Pampati V, Hirsch JA. Reversal of growth of utilization of interventional techniques in managing chronic pain in Medicare population post Affordable Care Act. Pain Physician 2017; 20:551-567.

45. Manchikanti L, Soin A, Mann DP, Bakshi S, Pampai V, Hirsch JA. Comparative analysis of utilization of epidural procedures in managing chronic pain in the Medicare population: Pre and post Affordable Care Act. Spine (Phila Pa 1976) 2019; 44:220-232.

46. Ovosi JO, Ibrahim MS, Bello-Ovosi BO. Randomized controlled trials: Ethical and scientific issues in the choice of placebo or active control. Ann Afr Med 2017; 16:97-100.

47. Sanapati J, Manchikanti L, Atluri S, et al. Do regenerative medicine therapies provide long-term relief in chronic low back pain: A systematic review and metaanalysis. Pain Physician 2018; 21:515-540.

48. Hong J, Jung S. Clinical effectiveness and prognostic indicators of parasagittal interlaminar epidural injection. Pain Physician 2016; 19:E877-E884. 
